\title{
Learning optics using a smart-phone
}

\section{Amparo Pons, Pascuala García-Martínez, Juan Barreiro, Ignacio Moreno}

Amparo Pons, Pascuala García-Martínez, Juan Carlos Barreiro, Ignacio Moreno, "Learning optics using a smart-phone," Proc. SPIE 9289, 12th Education and Training in Optics and Photonics Conference, 92892P (17 July 2014); doi: 10.1117/12.2070753

SPIE Event: 12th Education and Training in Optics and Photonics Conference, 2013, Porto, Portugal 


\title{
Learning Optics using a smart-phone
}

\author{
Amparo Pons ${ }^{1}$, Pascuala García-Martínez ${ }^{1}$, Juan Carlos Barreiro ${ }^{1}$ and Ignacio Moreno $^{2}$ \\ ${ }^{1}$ Departament d’Òptica, Universitat de València, 46100 Burjassot (Valencia), Spain. \\ ${ }^{2}$ Departamento de Ciencia de Materiales, Óptica y Tecnología Electrónica, Universidad Miguel Hernández de Elche, \\ 03202 Elche (Alicante), Spain.
}

\begin{abstract}
We propose several ideas about how to teach Optics using smart-phones. We think that the almost addictive interest of students in smart-phone technology can be useful for the benefits of learning. Moreover as all of our students are from university level, it helps that mostly all of them own a device. In this work we review many possibilities that using a smart-phone offer from the teaching point of view. We begin with a search of different apps about Optics. Then we also use the device as a sensor for implementing some experiments, we analyze accessories such as telescope and microscope lenses and finally, when the smart-phone is over, we use different parts to teach diffraction or imaging.
\end{abstract}

\section{INTRODUCTION}

Although most students spend huge amount of their time holding a smart-phone, few of them realize that they can learn Optics with it. Also for many educators, seeing smart-phones in their classroom is somehow annoying because students used them to be distracted. In order to stray such attention to productive learning subjects, we first propose to study different apps available for iOS, Android, and Windows based phones.

Besides apps, we might wonder what's inside a smart-phone about Optics? We can deconstruct such a device in order to explain geometrical optics, physical optics, photometry, optical materials, etc. We can learn the ability of such devices for imaging, by studying the type of lens they use. The connection between the size of the sensor (megapixels) and the focal length of the lens can be analyzed. Most smart-phones have quite small sensors, so focal lengths are short (less than $4 \mathrm{~mm}$ ) creating a wide-ranging depth of field. Another interesting issue to teach using the smart-phone is diffraction. We show the resolution limits of the imaging phone system. Regarding optical materials, we might teach liquid crystal (LC) technology based on nematic twisted LC used in a smart-phone in the display area.

In addition we review different accessories used to image magnification (as telescopes for instance) for clinical and medical purposes. Different optical demonstrations are also shown using the smart-phone.

The manuscript is organized as follows: in Section 2 we review different apps, mostly those free available, that cover many aspects in the area of Optics, such as geometrical optics, optometry, photography, photometry, visual optics, among others. In Section 3, we use different optical lens accessories for optical imaging. The possibility of measurement made by smart-phones is treated in Section 4. In Section 5 we use different pieces as camera, sensor, screen, etc. obtained by dismantle the smart-phone to teach diffraction or geometrical optics. Conclusions are shown in Section 6.

\section{DIFFERENT APPS ABOUT OPTICS}

When we combine modern smart-phone with wireless internet access and the remarkable number of cheap and free mobile apps now available, we find that they are truly amazing pocket-sized learning devices. Whether educators like them or hate them, the reality is that smart-phones are going to become pervasive in our classrooms, if they aren't already. We have found several apps with relative interest in optics, some of them are described in this section.

Optics Clinical Calculator [1] (Fig. 1(a)) is a vital tool for every general or refractive ophthalmologist, ophthalmology resident, optometrist, and optician. With Optics, you have streamlined, intuitive access to calculators used daily in prescribing lenses and understanding optics. 
APP for Learning Optics (AFLO) [2] (Fig. 1(b)) is a free APP that aims to provide simple knowledge of Optics for Physics. It consists of two sections: a Tutorial section and a Simulator section. In the Tutorial section, basic optical concepts are mentioned in text and image based table cells. Formulas are also included. In the Simulator section, optical simulators are provided such that students can verify the materials that they have studied. Users can visually study the effect by changing the parameters (such as lens focal length, object's distance or refractive index of glass) of the corresponding optical systems. It gives an idealized environment for student to perform optical experiments.

SPIE Field Guide for Geometrical Optics app [3] (Fig. 1(c)), derives from the treatment of geometrical optics that has evolved from both the undergraduate and graduate programs at the Optical Sciences Center at the University of Arizona. This app covers Gaussian imagery, paraxial optics, first-order optical system design, system examples, illumination, chromatic effects, and an introduction to aberrations. The appendices provide supplemental material on radiometry and photometry, the human eye, and several other topics.

Optical Illusions 100+ [4] (Fig. 1(d)) is a highly entertaining collection of more than 100 illusions, which does not require Internet connection. You can amuse yourself with illusions that appear to move, psychedelic patterns trick your eyes, and amazing illustrations that contain cool hidden images.

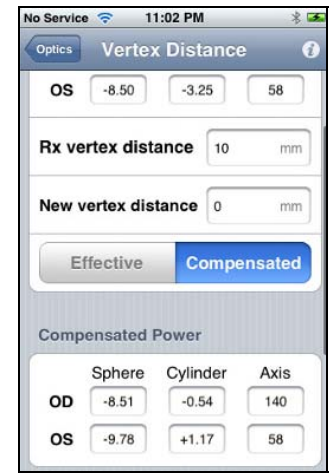

(a)

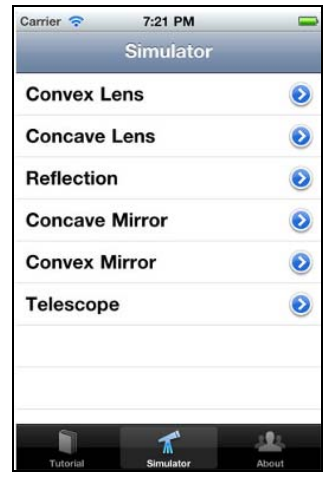

(b)

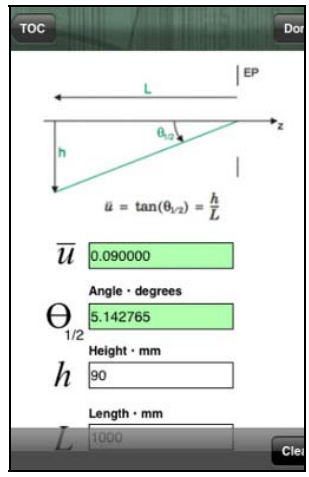

(c)

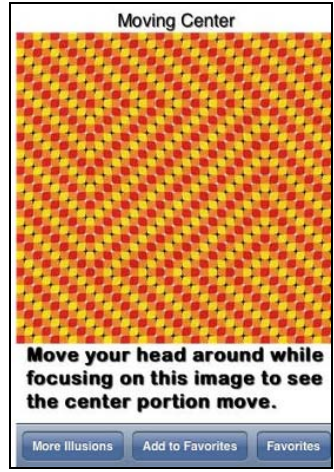

(d)

Figure 1. Different apps snapshots of several subjects in Optics

These apps can be used in the classroom where the students can run the apps in their smart-phones. They can make calculations and also visualizing many optical experiments to improve their understanding of the subjects.

\section{SMART-PHONE OPTICAL ACCESORIES}

One of the main uses of smart-phone is to take pictures. Nowadays due to platforms like Instagram apps, a photo-sharing tool that is having millions of followers around the world, the interest about photography is increasing. There are many apps designed specifically to process pictures and to transform them into beautiful creations. From an optics learning point of view, the interest of student about all this subjects can help the teachers to introduce the basis of photography and optical imaging.

Student must know that these pictures are captured with a small lens and matrix light sensor that provides the digital image to be visualized in the smart-phone display. The lens focal length is around a few millimeters, and the size of the sensor is typically around 4,5 $\mathrm{mm} \times 3,4 \mathrm{~mm}$. Also, the lens system is a certain combination of positive and negative refractive power surfaces to increase the resolution, the wide-angle and the aperture. The material use to build the lenses is typically polystyrene [5] and Zeonex E48R [6]. Certain knowledge of the sensor is also important for the student, since it is the final image registration device. In this case, parameters like the pixel size and the number of pixels are crucial. However from teaching point of view, we cannot forget that we cannot resolve pixels smaller than the Airy diffraction pattern. Again, physical optics is needed to understand imaging. 
But, although smart-phones camera system is enough to teach the basics of imaging, there is always room for improvement. Multiple optical accessories such as telescopes, microscopes, or other lens systems are available nowadays in the market. Those accessories are easily assembled to the smart-phone and with the appropriate installed apps the smart-phone can be very quickly turned into a medical instrument, measurement device or even into a scientific microscope or an astronomical telescope. As an example, Fig. 2 shows a smart-phone with an $8 \times$ lens accessory [7]. In Fig. 3 we show pictures taken with and without telescope accessory. Subjects like magnification and angular field can be very easily viewed with this system.

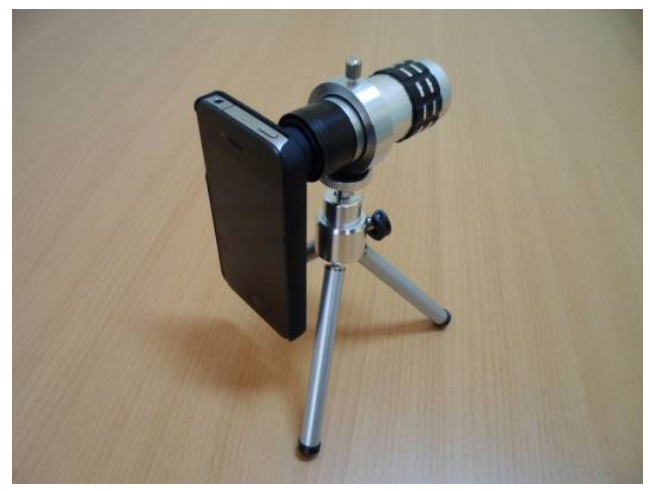

Fig. 2. Picture of a smart-phone with an $8 \times$ telescope accessory.

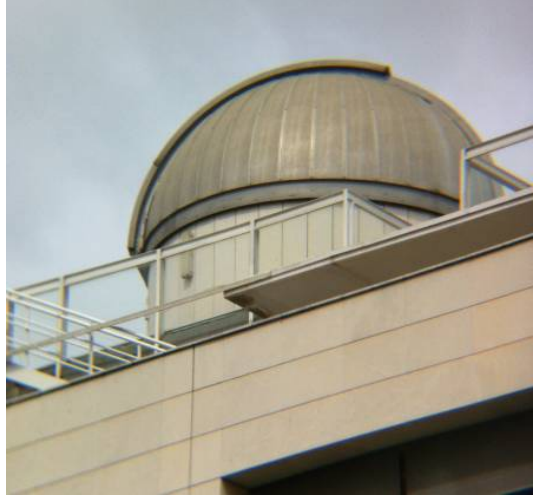

(a)

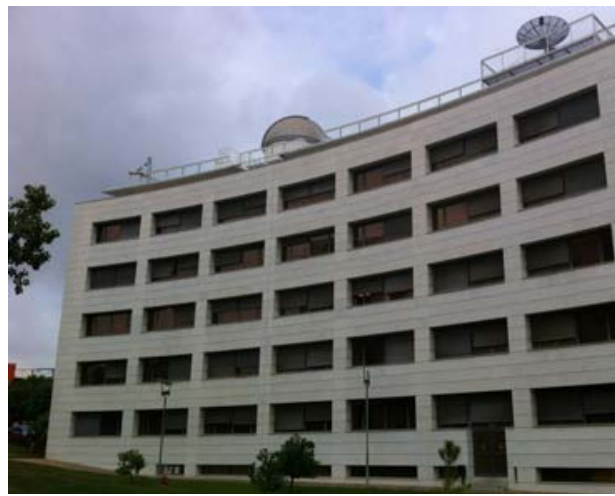

(b)

Fig. 3. Comparison between the image capture (a) with a smart-phone with added telescope lens accessory and (b) with only the conventional lens.

\section{OPTICAL EXPERIMENTS USING SMART-PHONES}

Another use of a smart-phone can be as a device to implement easy optical experiments in the classroom, so there is no need to move students to a laboratory with more complex equipment. Polarization can be explained since the majority of smart-phone displays emit linear polarized light. A simple photoelasticity experiment to observe the strain and stress in a transparent plastic material can be implemented just placing it between the display of the phone (acting as the source of input polarized light) and a common linear polarizer (serving as analyzer). If there is no stress on the plastic, the field of view behind the polarizer does not change the polarization of the light. But, this situation changes when the plastic is subjected to stress. Due to birefringence, the light is generally elliptically polarized as it passes through the plastic, i. e. it receives a component in the direction of the analyzer. Dark lines show the points where the locally occurring optical axis of the plastic happens to be aligned perpendicular to the analyzer transmission axis. Also bright areas show a colored structure, since different wavelength experience different phase shift when traversing the birefringent material (see Fig. 4). Note that, the picture of a white sheet of paper displayed in the smart-phone is used as a source of linear 
polarized white light, and no stress in the plastic material is observed without the analyzer (Fig. 4(a)). However, this situation dramatically changes when we place an analyzer with its polarization axis perpendicular to the smart-phone polarized light, and the brightly colored strain lines are observed (see Fig. 4(b)).

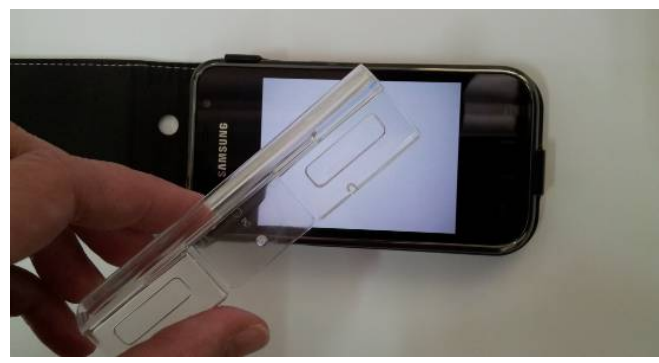

(a)

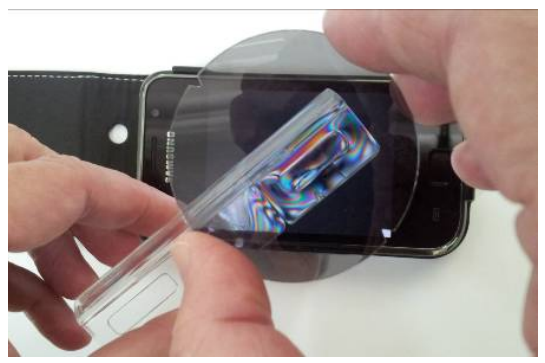

(b)

Fig. 4. (a) No strain lines are observed using a polarized light smart-phone and a plastic object. (b) Colored strain lines become clearly visible when we add the final analyzer.

Many other experiments and exercises can be taught to students in the classroom using the smart-phone. For instance, they can learn diffraction as the physical phenomenon that limits the resolution of their pictures, no matter how many megapixels their camera may have. Normally the diffraction effect is negligible, since smaller apertures often improve sharpness by minimizing lens aberrations. However, for sufficiently small apertures, the camera is diffraction limited and the pictures may be affected. For an ideal circular aperture, the 2-D diffraction pattern is the Airy disk, and its width, defined as the diameter of the first dark circle, $\mathrm{r}_{1}$, can be estimated as

$$
\mathrm{r}_{1}=1,22 \lambda \mathrm{f} / \# \quad,
$$

where $f / \#$ is the lens $f$ number and $\lambda$ is the illumination wavelength. Students can find it out by estimating the $f$ number of their smart-phone lens (or looking it on internet), and then calculating the size of the Airy disc. This easy calculation helps students to understand that diffraction thus sets a fundamental resolution limit that is independent of the number of megapixels, as frequently is mistakenly assumed.

\section{WHEN A SMART-PHONE IS OVER}

Previous ideas exits in the context of an operational smart-phone, however even when the device is damaged or finished it still can be useful for teaching optics using interesting pieces as the sensor, lens, or display. In fact the periodical structure of the pixelated sensor or display, can be used as a diffraction grating whose period will give us the size of the pixel.

The next experiment shows how we have used the display to calculate the size of the pixels in a smart-phone display [8]. In Fig. 5(a) we show the broken smart-phone. We have removed the display area (shown in Fig. 5(b)) and have taken a microscopic image of it, shown in Fig. 5(c), where it is apparent its grating-like structure. Note that in the figure are also clearly visible the three rectangular shaped primary chromatic components (red, green and blue) of each pixel. If we illuminate the grating with a coherent laser beam of wavelength $\lambda$, we will obtain its Fraunhofer diffraction pattern (Fig. 5(d)) at a observation plane located at a distance D large enough - in general, few meters of distance. According to the grating equation, the distance, $\mathrm{p}$, between the consecutive diffraction orders is given by

$$
\mathrm{p}=\lambda \mathrm{D} / \mathrm{d}
$$

where $\mathrm{d}$ is the distance between adjacent pixels (equal to size of the grating pixel, marked with a white square in Fig. 5(c)). Using Eq. (2), we have obtained that the size of the pixel is $173,8 \mu \mathrm{m} \times 174,4 \mu \mathrm{m}$, whereas the size measured using the microscope is $175,23 \mu \mathrm{m} \times 175,23 \mu \mathrm{m}$ [8]. 


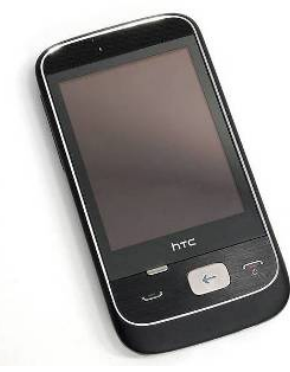

(a)

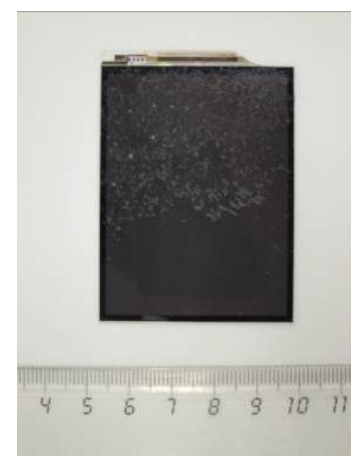

(b)

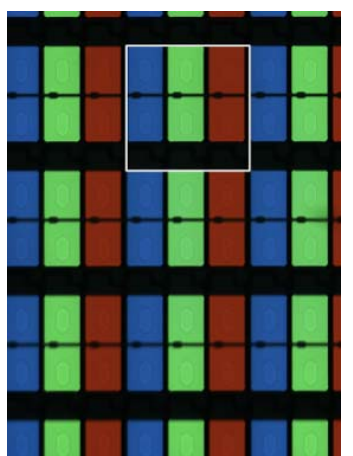

(c)

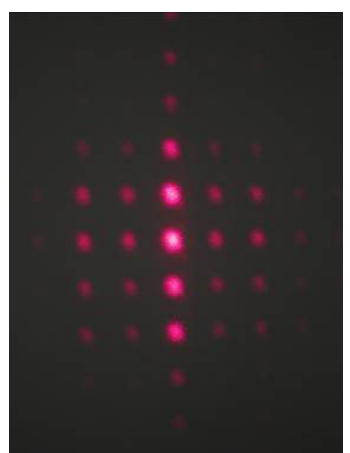

(d)

Fig. 5. (a) Smart-phone. (b) Color display extracted from the smart-phone, (c) Microscopic (10x) image of the Fig. 5(b) where a pixel's contour is highlighted with a white square, (d) diffraction pattern observed when the grating-like structure of Fig 5(c) is illuminated with a coherent red laser beam $(\lambda=650 \mathrm{~nm})$.

\section{CONCLUSIONS}

We have covered several Optics topics to be taught using a smart-phone. Nowadays most of the students have one, and they use it quite often without knowing that they can learn Optics with it. Diffraction, optical imaging, polarization, etc. can be easily explained taking advantage of the apps, and also of the physical properties of the smart-phone. In addition, when the smart-phone is damaged, several pieces can also serve to teach Physical Optics. We feel that using one of the most student attractive devices in the classroom will guarantee their interest on Optics.

\section{ACKNOWLEDGEMENTS}

This work was supported by the spanish Ministerio de Economía y Competitividad (Projects: FIS2012-39158-C02-02 and DPI2012-32994), the Generalitat Valenciana (Project: PROMETEO2009-077) and the Universitat de València (Project: GCID35/2009).

\section{REFERENCES}

1. Optics Clinical Calculator by Evan Schoenberg , M.D . (c) 2012 Regular Rate and Rhythm Software.

2. App for Learning Optics, by Department of Applied Physics, Hong Kong Polytechnic University (C) 2012 Department of Applied Physics (HKPolyU).

3. SPIE Field Guide for Geometrical Optics app. SPIE @ 2012 SPIE.

4. Optical Illusions $100+$ by Matthew King. ( $)$ Matthew King.

5. http://refractiveindex.info/?group=PLASTICS\&material=PS

6. http://refractiveindex.info/?group=PLASTICS\&material=ZeonexE48R

7. Ashley N. Paddock, "IPhone becomes multipurpose imaging tool”, Photonics Spectra, 25-16 (December 2011).

8. J. J. Barreiro, A. Pons, J. C. Barreiro, J. C. Castro-Palacio and J. A. Monsoriu, "Diffraction by electronic components of everyday use", American Journal of Physics (accepted). 